\section{An Update on Venous Thromboembolism in Cancer}

\author{
Michael B. Streiff, MD
}

This issue of JNCCN-Journal of the National Comprehensive Cancer Network focuses on one of the more common causes of morbidity and mortality in cancer patients: venous thromboembolism (VTE). VTE encompasses deep and superficial thrombosis of the extremities, as well as pulmonary embolism. Contemporary estimates note that VTE is the second most common cause of death in patients with cancer, after the cancer itself. ${ }^{1}$

Despite widespread recognition of the increased risk of VTE in patients with cancer and a growing list of therapeutic options for VTE prevention, prescription of VTE prophylaxis in the oncology setting remains low. In the IMPROVE registry, an international survey of VTE prophylaxis in medical inpatients, only $45 \%$ of patients with cancer received VTE prophylaxis. ${ }^{2}$ One reason for this apparent reluctance of providers may be a lack of data supporting the efficacy of VTE prophylaxis in hospitalized medical oncology patients. No randomized controlled trials studying VTE prophylaxis in medical oncology inpatients have been conducted, and randomized controlled trials in medical inpatients have included a minority of patients with cancer. ${ }^{3-5}$ Conversely, surgical oncology patients have been studied in a number of randomized clinical trials, which have supported the efficacy of VTE prophylaxis in this population..$^{6-8}$ The availability of this published evidence may explain the greater adherence to prophylaxis seen in the FRONTLINE and IMPROVE studies. ${ }^{2,9}$

Another potential factor decreasing the acceptance of VTE prophylaxis in medical oncology patients is that most events occur in the outpatient setting. Although several recent, well-designed, randomized controlled trials, including PROTECHT and SAVE-ONCO, have shown statistically significant reductions in VTE with routine prophylaxis, ${ }^{10,11}$ these results have not resulted in a change of clinical practice. Other factors contributing to this lack of acceptance of routine VTE prophylaxis in ambulatory patients with cancer include low event rates, an absence of a mortality benefit, and the need for parenteral administration.

The obvious solution is to identify patients whose risk of VTE is high enough to warrant the risks and inconveniences of VTE prophylaxis. In this issue of JNCCN, Alok Khorana, MD, a leading authority on VTE risk stratification and prevention in patients with cancer, provides an overview of randomized clinical trials of VTE prophylaxis in ambulatory patients with cancer, VTE risk assessment in outpatients with cancer, current guideline recommendations, and potential future directions in this important area of research.

On October 19, 2010, dabigatran etexilate (Pradaxa; Boehringer-Ingelheim, Ingelheim, Germany) was approved by the FDA for thromboprophylaxis of nonvalvular atrial fibrillation. As it is the first nonvitamin $\mathrm{K}$ antagonist oral anticoagulant, its approval, not surprisingly, was marked by unprecedented enthusiasm among physicians and patients. Two oral direct factor Xa inhibitors, rivaroxaban (Xarelto; Janssen Pharmaceuticals, Raritan, NJ) and apixaban (Eliquis; Bristol-Myers Squibb, Princeton, NJ) have subsequently been approved for thromboprophylaxis of nonvalvular atrial fibrillation and VTE after hip or knee arthroplasty, and treatment of VTE and thromboprophylaxis of nonvalvular atrial fibrillation, respectively. A number of other novel oral anticoagulants are in different stages of clinical investigation. Because parenteral agents, particularly low-molecular-weight heparin, are favored for prevention and treatment of VTE in patients with cancer, tremendous interest has been seen in using these new oral agents in the cancer setting. But do

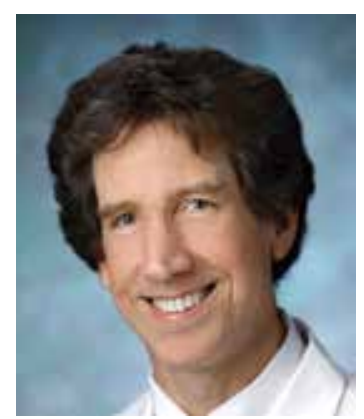

Michael B. Streiff, MD

Michael B. Streiff, MD, is Associate Professor of Medicine at The Sidney Kimmel Comprehensive Cancer Center at Johns Hopkins, and the Chair of the NCCN Guidelines Panel on Venous Thromboembolic Disease.
The ideas and viewpoints expressed in this editorial are those of the author and do not necessarily represent any policy, position, or program of NCCN. 
we know enough about novel oral anticoagulants to use them for our patients? Also in this issue of JNCCN, Paul Hendrie, MD, and David Garcia, MD, from the Fred Hutchinson Cancer Research Center/Seattle Cancer Care Alliance at the University of Washington review the published randomized clinical trials on novel oral anticoagulants and provide an evidence-based answer to this important and timely question.

VTE remains one of the most common complications faced by patients with cancer. Critical to improving the outcome of these patients with VTE is identifying patiesnts with cancer at risk for VTE and establishing the most effective approaches to management. The articles in this issue of JNCCN demonstrate that the science of treating VTE in patients with cancer has come a long way, but much work remains to be done to improve outcomes.

\section{References}

1. Khorana AA, Francis CW, Culakova E, et al. Thromboembolism is a leading cause of death in cancer patients receiving outpatient chemotherapy. J Thromb Haemost 2007;5:632-634.

2. Tapson VF, Decousus H, Pini M, et al. Venous thromboembolism prophylaxis in acutely ill hospitalized medical patients: findings from the international medical prevention registry on venous thromboembolism. Chest 2007;132:936-945.

3. Samama MM, Cohen AT, Darmon JY, et al. A comparison of enoxaparin with placebo for the prevention of venous thromboembolism in acutely ill medical patients: prophylaxis in Medical Patients with Enoxaparin Study Group. N Engl J Med 1999;341:793-800.

4. Leizorovicz A, Cohen AT, Turpie AG, et al. Randomized, placebo-controlled trial of dalteparin for the prevention of venous thromboembolism in acutely ill medical patients. Circulation 2004;110:874879.

5. Cohen AT, Davidson BL, Gallus AS, et al. Efficacy and safety of fondaparinux for the prevention of venous thromboembolism in older acute medical patients: randomised placebo controlled trial. BMJ 2006;332:325-329.

6. Bergqvist D, Agnelli G, Cohen AT, et al. Duration of prophylaxis against venous thromboembolism with enoxaparin after surgery for cancer. N Engl J Med 2002;346:975-980.

7. Efficacy and safety of enoxaparin versus unfractionated heparin for prevention of deep vein thrombosis in elective cancer surgery: a double-blind randomized multicentre trial with venographic assessment. ENOXACAN Study Group. Br J Surg 1997;84:1099-1103.

8. Rasmussen MS, Jorgensen LN, Wille-Jorgensen P, et al. Prolonged prophylaxis with dalteparin to prevent late thromboembolic complications in patients undergoing major abdominal surgery: a multicenter randomized open-label study. J Thromb Haemost 2006;4:2384-2390.

9. Kakkar AK, Levine M, Pinedo HM, et al. Venous thrombosis in cancer patients: insights from the FRONTLINE survey. Oncologist 2003;8:381-388.

10. Agnelli G, Gussoni G, Bianchini C, et al. Nadroparin for the prevention of thromboembolic events in ambulatory patients with metastatic or locally advanced solid cancer receiving chemotherapy: a randomised, placebo-controlled, double-blind study. Lancet Oncol 2009;10:943-949.

11. Agnelli G, George DJ, Kakkar AK, et al. Semuloparin for thromboprophylaxis in patients receiving chemotherapy for cancer. N Engl J Med 2012;366:601-609. 